\title{
CARACTERIZACIÓN DE ACCIDENTES DE TRABAJO POR CAÍDA DE OBJETOS EN EMPRESAS DEL SECTOR HIDROCARBUROS
}

\author{
Gloria A. Jaramillo \\ Universidad Cooperativa de \\ Colombia Neiva \\ Andreajh6@hotmail.com
}

\author{
Iván Darío Roa \\ Universidad Cooperativa de \\ Colombia Neiva \\ ivancho_3420@hotmail.com
}

\author{
Jairo Arturo Motta \\ Universidad Cooperativa de \\ Colombia Neiva \\ mottayo@hotmail.com
}

\author{
Tatiana F. García Ríos \\ Universidad Cooperativa de Colombia Neiva \\ Tatiana.garciar@campusucc.edu.co
}

\author{
Alfredis González Hernández \\ Universidad Cooperativa de Colombia Neiva \\ alfredis.gonzalezh@campusucc.edu.co
}

(Tipo de Artículo: Investigación. Recibido el 20/11/2014. Aprobado el 12/12/2014)

\begin{abstract}
RESUMEN
En una multinacional del sector de hidrocarburos prestadora de servicios de perforación y workover, que opera en las zonas de Tolima y Santander Colombia, se pudo desarrollar una investigación con el objetivo de describir la situación de accidentalidad causada por caída de objetos que se presenta en el área de taladros de perforación. La información se recolectó de los reportes de accidentes e incidentes que reposaban en la base de operaciones en Sopó entre el periodo 2010 y 2011 . Al analizar los hallazgos se pudo evidenciar que son cuatro los agentes causales más significativos, dentro de los cuales están los denominados Golpes por objetos, ocupando un 68\%; atrapado por o entre un objeto con $41 \%$; caída de personas con un 30\% y caída de objetos con $21 \%$. Este último hallazgo se deriva por la actividad propia de la perforación por la manipulación de llaves hidráulicas, objetos adicionales de acero, martillos, entre otros y su causa básica fue la falta de supervisión, liderazgo y/o planificación con $39 \%$, seguida de procedimientos de trabajo inadecuados con el $22 \%$ y el mantenimiento inadecuado con el $11 \%$, lo que concluye que en su mayoría son errores humanos.
\end{abstract}

Palabras clave. Accidentes laborales, actividades de alto riesgo, caída de objetos, incidentes, mortalidad en el trabajo.

\section{CHARACTERIZATION OF WORKPLACE ACCIDENTS FROM FALLING OBJECTS IN OIL SECTOR COMPANIES}

\begin{abstract}
In a multinational of hydrocarbon sector provider of drilling and workover services, which operates in the region of Tolima and Santander in Colombia, a research was conducted with the purpose of describing the accidents caused by falling objects in the borehole zone. The information was collected from the reports of accidents and incidents of Sopó's base of operations in 20102011 periods. After analyzing the findings it was evident that there were four significant causative agents, we have the so-called struck by objects with 68\%; caught by or between an object with $41 \%$; falling people with $30 \%$ and falling objects with $21 \%$. This latter finding is derived from the activity of drilling by manipulating hydraulic wrenches, additional objects of steel and hammer stems, among others, and the basic cause was the lack of supervision, leadership and / or planning with $39 \%$, followed by inadequate work procedures with $22 \%$ and poorly maintenance with $11 \%$ from which we conclude that most of them are derived from human errors.
\end{abstract}

Keywords. Workplace Accidents, High-Risk Activities, Falling objects, Incidents, Death at work.

\section{Caractérisation d'accidents du travail à cause des chutes d'objets dans entreprises du secteur d'hydrocarbures}

\begin{abstract}
Résumé
Dans une multinationale du secteur d'hydrocarbures qui réalise forage et workover qui opère dans les régions de Tolima et Santander dans la Colombie, on a réalise une recherche avec l'objectif de décrire les accidents à cause des chutes d'objets dans la zone des perceuses. L'information est collectée des reports d'accidents et incidents de la base d'opérations dans Sopó entre le période 2010 et 2011. D'après l'analyse des trouvailles on a vérifié qu'il y a quatre agents causales les plus significatifs, en trouvant les coups pour objets avec le $68 \%$, attrapé pour ou entre un objet avec $41 \%$, chutes des personnes avec $30 \%$ et chutes d'objets avec $21 \%$. La dernière trouvaille est dérivée de l'activité der forage par la manipulation des clés hydrauliques, objets d'acier, marteaux piqueurs, entre autres, et son cause principale a été le manque de supervision, leadership et planification avec $39 \%$, procédures de travail inadéquates avec $22 \%$ et maintenance inadéquat avec $11 \%$, ce qui résulte en une majorité d'erreurs humaines.
\end{abstract}

Mots-clés. Accidents de travail, activités de haut risque, chutes d'objets, incidents, mortalité dans le travail. 


\section{INTRODUCCIÓN}

La ley 1562 del 2012 en su Art.3, define Accidente de trabajo como: " todo evento no deseado que sobrevenga por causa o con ocasión del trabajo y que en el trabajador genere una lesión orgánica, una perturbación funcional o psiquiátrica, una invalidez o la muerte" [15]; concepto que al ser relacionado con el objeto de investigación, confirma que las lesiones causadas a los operadores por caídas de objetos en alturas es un tipo de accidente de trabajo que se presenta con frecuencia en empresas de hidrocarburos al momento de realizar tareas como reacondicionamiento pozos, armar taladros de perforación, extracción de petróleo o gas, operación con grúa y el mantenimiento de equipos y maquinas.

La gravedad de las lesiones que un trabajador puede sufrir a causa de la caída de objetos, dependen en gran manera del tamaño del objeto y la altura. Lo que indica que si se dejan caer objetos desde alturas máximas (como las manejadas en las zonas de perforación de taladro que oscilan entre 90 y 120 pies aproximadamente), este podría originar lesiones fatales o pérdidas corporales significativas en los empleados que están expuestos a estos riesgos laborales. Dicha situación, demandan el uso adecuado y continuo de elementos de protección personal y cuidados específicos al momento de adecuar la zona para realizar los trabajos.

La investigación también arrojo que fueron 28 incidentes laborales generados por caídas de objetos, lo que implica que se deben tomar medidas preventivas tales como: capacitaciones diarias, descripción detalladas de las labores a realizar en el día, y además proporcionar información detallada del equipo a manipular que se le entrega a los operarios a través de manuales y fichas técnicas, para evitar que se presenten accidentes de trabajo.

Al retomar el concepto de "Incidente" se puede ver que es un "suceso o sucesos relacionados con el trabajo en el cual ocurre o podría haber ocurrido un daño, o deterioro de la salud ( $\sin$ tener en cuenta la gravedad), o una fatalidad" (OHSAS 18001:2007), concepto que ayuda a detectar los puntos débiles o controles operacionales fallidos para potenciar medidas correctivas y evitar su repetición.

Por lo anterior el estado colombiano a través de La Ley 9 de 1979 "dicta medidas sanitarias mediante la cual se hace responsable del control y vigilancia de Sistema General de Riesgos Profesionales y hace énfasis en la resolución 3673 de 2008 en relación al desarrollo de trabajo en alturas [14], el cual en su artículo 10 "Item 3.1" cita los requerimientos generales para evitar accidentes de trabajo por caída de objetos.

De tal modo investigaciones a nivel nacional como la GUÍA PARA LA APLICACIÓN DEL SISTEMA DE PREVENCIÓN DE CAÍDA DE OBJETOS EN EQUIPOS DE PERFORACIÓN "DROPs" sirven como referencia, puesto que propone métodos e indicaciones lo cual le permite a la empresas del sector que no se generen lesiones o muertes a causa de un objeto que cae desde la torre. En resumen indica las causas por las cuales un objeto pequeño que cae desde una gran altura puede traer consigo consecuencias letales [12].

Otro estudio a nivel nacional es el denominado DISEÑO DE UN PROGRAMA PARA LA PREVENCIÓN DE CAÍDA DE OBJETOS EN TALADROS DE PERFORACIÓN, hace una priorización para la toma de medidas correctivas y preventivas de caídas de objetos por causa de la actividad laboral en taladros de perforación, se inició con el análisis de accidentes ya ocurridos desde diferentes alturas de taladros de todo el mundo donde el $100 \%$ de los objetos caídos analizados, el $46 \%$ corresponde a objetos que son instalados y dejados fijos en la torre durante todo el trabajo de perforación de un pozo, y el $54 \%$ corresponde a objetos que se deben instalar o son subidos y manipulados por el hombre en altura durante determinada actividad. En cuanto a la causa básica se especifica que tienen que ver primero con un $32 \%$ por ingeniería inadecuada de los equipos o herramientas, seguido por un $28 \%$ relacionado con estándares deficientes de trabajo; es decir, no existen o no son adecuados los 91 procedimientos de trabajo, y en el tercer lugar con un $24 \%$ se encuentran las causas relacionadas con el uso y desgaste de los equipos; en esta naturaleza todas las causas encontradas tienen que ver con fallas en los procesos de inspección y control deficiente [7].

Como antecedentes a nivel internacional se tomó el documento investigativo "CONDICIONES DE VIDA Y DE TRABAJO EN LA ACTIVIDAD PETROLERA EN LA PROVINCIA DE NEUQUÉN", que hace alusión a la identificación de los riesgos laborales en el sector de hidrocarburos, la definición caídas de objetos por desplome, caídas de objetos en manipulación, caídas de objetos desprendidos, que al evaluarlos y valorarlos se relacionan con el trabajo de alturas y se llega a la conclusión que la siniestralidad de los peligros significativos no quedan en reportes estadísticos, ya que los únicos accidentes laborales que se relacionan con las productoras petroleras son los mortales, por el impacto mediático [3].

\section{DESARROLLO DEL ARTÍCULO}

\subsection{Materiales y Métodos}

Esta investigación es de enfoque cuantitativo, diseño no experimental, de corte transversal descriptivo, al pretender caracterizar los accidentes de trabajo por causa de caída de objetos mediante la descripción de las variables agentes de lesión, áreas y causas de ocurrencia.

Se tomó como población los incidentes y accidentes reportados para el periodo 2010-2011 que ocurrieron en los 228 taladros de la compañía y se tuvo como muestra los incidentes y accidentes reportados para los años 2010 y 2011 (308 eventos en total), que tuvieron 
Ing. USBMed, Vol. 5, No. 2, Julio-Diciembre 2014

ocurrencia en los 19 taladros de perforación y 8 taladros workover de pozos petroleros con un total de 27 taladros que componen la flota terrestre de la base Sopó.

La investigación se realizó mediante 4 fases.

Fase I. Revisión de referencia bibliográfica: sobre accidentalidad oficialmente reportada por compañías de servicios de perforación y workover de la industria de hidrocarburos a nivel internacional, nacional y local.

Fase II. Caracterización: Se caracterizan los accidentes de trabajo reportados en los periodos revisado por agentes causante de lesión en una compañía ubicada en la región del Alto Magdalena (Tolima) y Magdalena Medio (Santander) y se realiza revisión de las investigaciones de accidentes de trabajo por causa de caída de objetos en el periodo de análisis, caracterizando las principales causas de ocurrencia de los mismos.

Fase III. Identificación: Identificación de peligros mediante la realización de un inventario donde se listará cualquier objeto que tenga el potencial de caer en las áreas en que se zonificó el taladro.

Fase IV. Análisis: La información recopilada fue procesada utilizando la herramienta microsoft excel 2010, se cuantificó con frecuencias simples y porcentajes de las variables de los datos obtenidos en la revisión y análisis de los accidentes reportados durante los periodos 2010 y 2011.

Los instrumentos para la información obtenida en los ejercicios de campo para la identificación de peligros y el levantamiento del inventario donde se listan los objetos que tengan potencial de caída y causen incidentes y/o accidentes en el lugar de trabajo, es la versión mejorada del formato 02-01-100-F002 - matriz de evaluación de riesgos del SIG de la compañía de la Guía Técnica Colombiana GTC 45 - Guía para la identificación de los peligros y la valoración de los riesgos en seguridad y salud ocupacional - Versión 2010 [12].

\subsection{Resultados}

Dentro del análisis que se realiza en la investigación en la Tabla 1 los accidentes reportados para la empresa en estudio, durante el periodo de tiempo en el año 2010 - 2011 los agente causales más significativos son los denominados Golpes por objetos con 63 accidentes reportados ocupando un $68 \%$, atrapado por o entre un objeto con 37 accidentes reportados ocupando un $41 \%$, caída de personas con 27 accidentes y un porcentaje de $30 \%$, y caída de objetos con 20 accidentes reportados ocupando un $21 \%$, este último ocupa un cuarto lugar y se observa que la tendencia en los agentes causales de lesión se mantiene durante los periodos caracterizados. Como se observa en la Fig. 1.
Tabla 1. Accidentes de trabajos por agente de lesión 2010 y 2011

\begin{tabular}{l|c|c}
$\begin{array}{c}\text { Accidentes de trabajos por } \\
\text { agente de lesión } \mathbf{2 0 1 0}\end{array}$ & $\begin{array}{c}\text { Año } \\
\mathbf{2 0 1 0}\end{array}$ & $\begin{array}{c}\text { Año } \\
\mathbf{2 0 1 1}\end{array}$ \\
\hline Caídas de personas & $14 \%$ & $16 \%$ \\
\hline Caídas de objetos & $12 \%$ & $9 \%$ \\
\hline $\begin{array}{l}\text { Golpes por objetos, a } \\
\text { excepción de caídas de objetos }\end{array}$ & $36 \%$ & $32 \%$ \\
\hline $\begin{array}{l}\text { Atrapado por o entre un objeto } \\
\text { Esfuerzos excesivos }\end{array}$ & $20 \%$ & $21 \%$ \\
\hline $\begin{array}{l}\text { Exposición a temperaturas } \\
\text { extremas }\end{array}$ & $4 \%$ & $1 \%$ \\
\hline $\begin{array}{l}\text { Exposición a la corriente } \\
\text { eléctrica }\end{array}$ & $0 \%$ & $1 \%$ \\
\hline $\begin{array}{l}\text { Exposición a sustancias } \\
\text { nocivas }\end{array}$ & $1 \%$ & $3 \%$ \\
\hline $\begin{array}{l}\text { Otras formas de accidente } \\
\text { a }\end{array}$ & $4 \%$ & $7 \%$
\end{tabular}

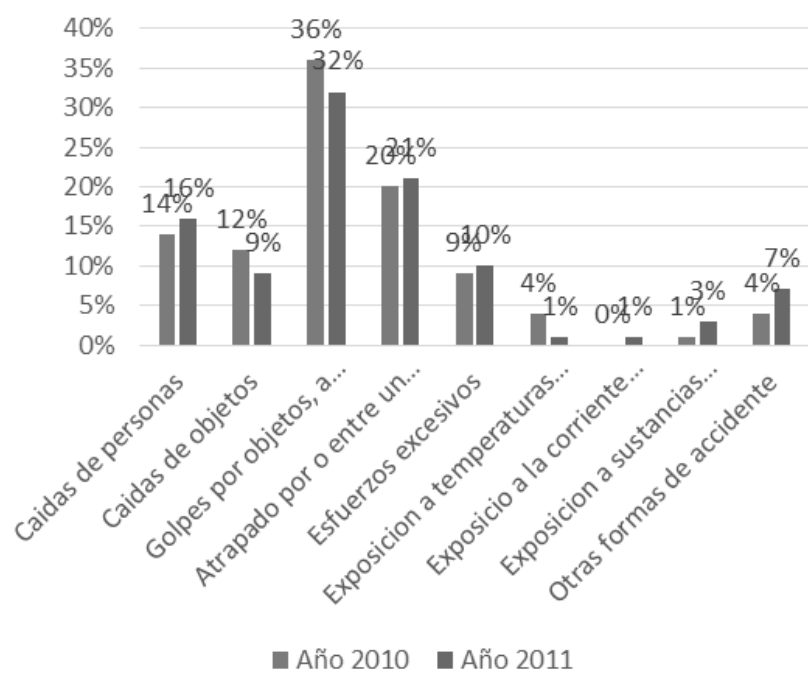

Fig. 1 Accidentes de trabajos por agente de lesión 2010-2011

Es así como se realizó un ejercicio en la caracterización de los accidentes de trabajo sumando a éstos los incidentes reportados en cada periodo (2010 - 2011) ya que los incidentes que no generaron lesiones a los trabajadores, tuvieron y tienen el potencial de causar serias lesiones y daños materiales, si las condiciones de su ocurrencia hubieran sido un tanto diferentes; donde se obtuvo una variación considerable en la tendencia mantenida y fue así como el agente causal de lesiones de mayor importancia continuo siendo la categoría golpes, pisadas, choques sobre, contra o por objetos, a excepción de caídas de objetos, que representa un 30\%, la categoría caídas de objetos pasó al segundo lugar en importancia como agente causal de lesiones con 48 incidentes reportados que representa un $23 \%$; al tercer lugar fue desplazada la categoría atrapado por / entre un objeto, con 37 accidentes de trabajo reportados, representado un $18 \%$ $y$ en el cuarto lugar como agente causal de lesiones quedó la categoría caídas de personas con 27 accidentes de trabajo reportados, presentando un $13 \%$ 
en la accidentalidad, como se observa en la Fig. 2.

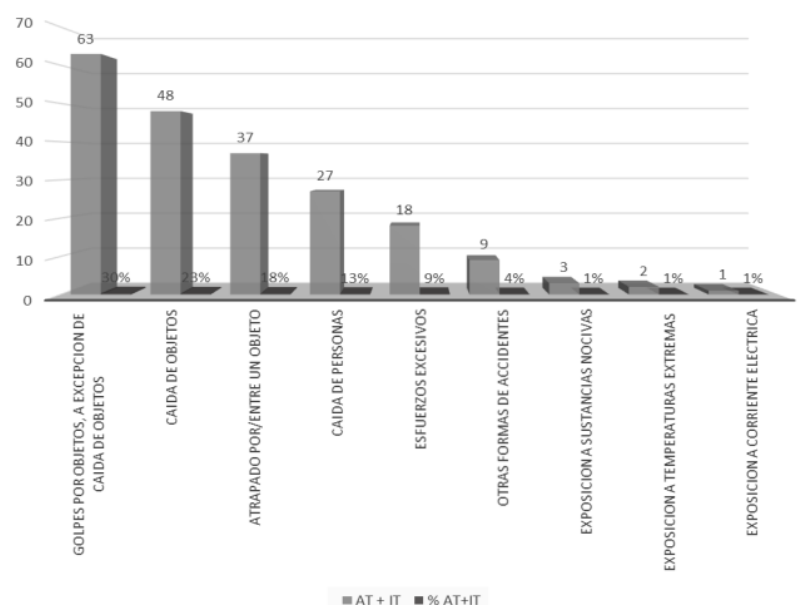

Fig. 2 Accidentes de trabajo por agente de lesión año 2010-2011 sumando incidentes potenciales de caídas de objetos.

Realizando un acumulado de los accidentes (AT) e incidentes de trabajo (IT) por el agente causal, en la Tabla 2 caída de objetos por zonificación del taladros para los periodos revisados 2010 - 2011 (20 en total), tenemos que la sección VII - Otras áreas del taladro con 11 accidentes reportados, representando un 55\% del total, seguido de la sección $\mathrm{V}$ - piso de la mesa rotaria con 4 accidentes reportados y el $20 \%$, las secciones IV - Top Drive / Bloque viajero y la sección VI - subestructura / BOP con dos accidentes de trabajo reportados cada una, comparten el tercer y cuarto lugar con el $10 \%$. En el quinto lugar encontramos a la sección III - Mástil / debajo del trabajadero con un accidente de trabajo reportado y el $5 \%$ del total. Para la caracterización de los incidentes en el año 2010-2011 (28 en total) ,tenemos que del total del $71 \%$ de los incidentes reportados ocurrieron en la sección VII Otras áreas del taladro con 20 incidentes, seguido con un $14 \%$ de la sección $\mathrm{VI}$ - subestructura / BOP con 4 incidentes, en tercer lugar encontramos la sección $\mathrm{V}$ piso de la mesa rotaria con dos incidentes y los lugares cuarto y quinto las secciones I corona y IV Top Drive / Bloque viajero con un incidente cada uno y un $4 \%$. Las secciones II - Trabajadero de tubería y III - Mástil / debajo del trabajadero no reportador incidentes por causa de caída de objetos durante el periodo de análisis. Como puede verse en la Fig. 3
Tabla 2. Caídas de objetos por zonificación taladros

\begin{tabular}{l|c|c}
$\begin{array}{l}\text { Caídas de objetos por } \\
\text { zonificación taladros }\end{array}$ & $\begin{array}{c}\text { AT Año } \\
\mathbf{2 0 1 0}- \\
\mathbf{2 0 1 1}\end{array}$ & $\begin{array}{l}\text { IT Año } \\
\mathbf{2 0 1 0} \\
\mathbf{2 0 1 1}\end{array}$ \\
\hline $\begin{array}{l}\text { I-Sección Coronaria } \\
\begin{array}{l}\text { II-Sección Trabajadero } \\
\text { tubería }\end{array}\end{array}$ & $0 \%$ & $4 \%$ \\
\hline $\begin{array}{l}\text { IlI-Sección Mástil /debajo del } \\
\text { trabajadero }\end{array}$ & $5 \%$ & $0 \%$ \\
\hline $\begin{array}{l}\text { IV-Sección Top Drive/Bloqueo } \\
\text { viajero }\end{array}$ & $10 \%$ & $4 \%$ \\
\hline $\begin{array}{l}\text { V-Sección piso de la mesa } \\
\text { rotatoria }\end{array}$ & $20 \%$ & $7 \%$ \\
\hline $\begin{array}{l}\text { VI-Sección subestructura } \\
\text { NOP }\end{array}$ & $10 \%$ & $14 \%$ \\
\hline $\begin{array}{l}\text { VII-Sección otras áreas del } \\
\text { taladro }\end{array}$ & $55 \%$ & $71 \%$
\end{tabular}

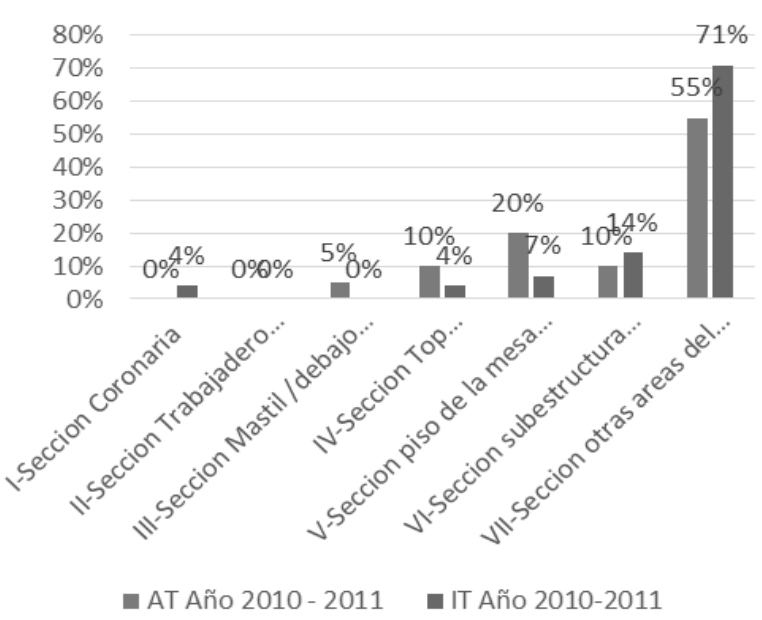

Fig. 3 Caídas de objetos por zonificación taladros.

Realizando una comparación de los accidentes e incidentes por caídas de objetos por zonificación del taladro se evidencia que la sección VII otras áreas representan una área critica puesto que para los años 2010 -2011 fueron donde más se reportaron tanto accidentes los cuales fueron 11 representando un 55\% e incidentes que fueron 20 representando un $71 \%$ el cual indica que es un área a intervenir.

Se utilizó como referente la clasificación del listado global de causas básicas / raíz del procedimiento 0201-109-F002, fecha de emisión 17 enero 2012. Revisión 10 del Sistema Integrado de Gestión de la compañía. Este listado global de causas básicas, posee nueve posibles causas agrupadas en dos categorías - Factores personales y Factores del Trabajo Teniendo en cuenta que las posibles causas de ocurrencia de los accidentes e incidentes por caídas de objetos obedecen a los criterios de los investigadores se optó por caracterizar éstos por sus causas intrínsecas sin considerar el área de ocurrencia. Se consideraron hasta tres causas básicas por accidente e incidente para facilitar la caracterización. Algunas investigaciones solo consideraron una sola causa básica en su elaboración. 
En la Tabla 3 causas básicas de caída de objetos, los accidentes de trabajo reportados en los periodos 2010 - 2011 sumaron 20 en total, se obtuvieron 44 causas básicas dentro de las cuales la causa falta de supervisión, liderazgo y/o planificación, con 18 selecciones quedo en el primer lugar con el $41 \%$. La causa procedimientos / estándares de trabajo inadecuados, con 7 selecciones en el segundo lugar con $16 \%$. En el tercer lugar de causas de ocurrencia de accidentes de trabajo por caída de objetos tenemos la causa diseño y/o mantenimiento inadecuados, con 5 selecciones y el $12 \%$ al igual que la causa comunicación inadecuada, con 5 selecciones y un 12\% en porcentaje. En cuanto a los incidentes para los periodos 2010-2011 sumaron 28 en total, se obtuvieron 65 causas básicas dentro de las cuales la causa falta de supervisión, liderazgo y/o planificación, con 25 selecciones quedo en el primer lugar con el $38 \%$. La causa procedimientos / estándares de trabajo inadecuados, con 17 selecciones en el segundo lugar con $26 \%$. En el tercer lugar de causas de ocurrencia de accidentes de trabajo por caída de objetos continua la causa ingeniería, diseño y/o mantenimiento inadecuados, con 7 selecciones y el $11 \%$, El cuarto y quinto lugar en causalidad lo comparten las causas comunicación inadecuada y abastecimiento y control de productos y servicios con 4 selecciones y un $6 \%$ cada una respectivamente. En la Fig. 4 puede observase los las causas básicas para accidentes e incidentes de trabajo en el periodo de tiempo 2010 -2011

Tabla 3. Causas básicas de caída de objetos

\begin{tabular}{|c|c|c|}
\hline $\begin{array}{l}\text { CAUSAS BASICAS DE } \\
\text { CAIDA DE OBJETOS }\end{array}$ & $\begin{array}{c}\text { AT Año } \\
2010-2011\end{array}$ & $\begin{array}{l}\text { IT Año } \\
2010-2011\end{array}$ \\
\hline $\begin{array}{ll}\text { Capacidad física/Mental } \\
\text { inadecuada }\end{array}$ & $2 \%$ & $0 \%$ \\
\hline $\begin{array}{c}\text { Comportamiento } \\
\text { /Motivación inadecuada }\end{array}$ & $2 \%$ & $3 \%$ \\
\hline $\begin{array}{ccc}\begin{array}{c}\text { Nivel } \\
\text { deficiente }\end{array} & \text { de } & \text { Habilidad } \\
\end{array}$ & $5 \%$ & $3 \%$ \\
\hline $\begin{array}{cc}\text { Falta } & \text { de } \\
\text { conocimiento/Capacitación }\end{array}$ & $7 \%$ & $5 \%$ \\
\hline $\begin{array}{l}\text { Fallas de supervisión, } \\
\text { liderazgo Y/o planificación }\end{array}$ & $41 \%$ & $38 \%$ \\
\hline $\begin{array}{c}\text { Ingeniería, diseño y/o } \\
\text { mantenimiento inadecuado }\end{array}$ & $12 \%$ & $11 \%$ \\
\hline $\begin{array}{l}\text { Abastecimiento } \\
\text { control de productos } y \\
\text { servicios }\end{array}$ & $2 \%$ & $6 \%$ \\
\hline \begin{tabular}{l}
\multicolumn{3}{c}{ Procedimientos/estánda } \\
res de \\
inadecuados
\end{tabular} & $16 \%$ & $26 \%$ \\
\hline $\begin{array}{l}\text { Comunicación } \\
\text { inadecuada }\end{array}$ & $11 \%$ & $6 \%$ \\
\hline reglas organizacionales & $2 \%$ & $2 \%$ \\
\hline
\end{tabular}

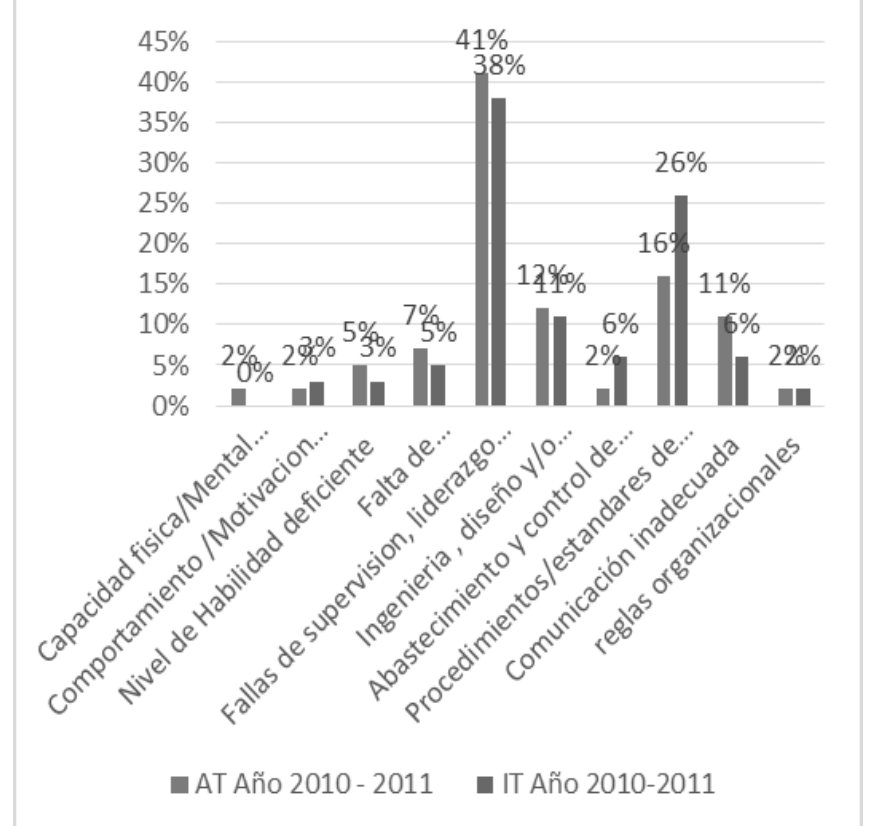

Fig. 4 Causas básicas de accidentes e incidentes de trabajo para periodo 2010-2011.

De lo anterior se puede llegar a la conclusión final que la causa básica o raíz de mayor selección durante la caracterización realizada fue la falta de supervisión, liderazgo y/o planificación con 39\%, seguida de procedimientos / estándares de trabajo inadecuados con el $22 \%$ y la ingeniería, diseño y/o mantenimiento inadecuados con el $11 \%$, por lo que se puede llegar a concluir que la en su mayoría son errores humanos.

\section{TRABAJOS FUTUROS}

Son muchas las alertas de seguridad generadas a lo largo del desarrollo de las actividades en la industria de hidrocarburos, en especial de empresas cuya actividad económica es la perforación y reacondicionamiento de pozos, muchos trabajadores son involucrados en accidentes por caída de objetos, lo cual genera pérdidas de vidas humanas y económicas. Por lo tanto este estudio descriptivo sirve como referente, ya que en el país son pocos los estudios investigativos realizados y relacionados con el tema; además sirve como dato estadístico en cuanto a la autoevaluación de accidentes e incidentes reportados, lo cual conlleva a un análisis crítico sobre la eficacia de la aplicación de medidas preventivas y de protección y planes de contingencia que se acogen y empelan, pero no son $100 \%$ efectivos para las empresas.

\section{CONCLUSIONES}

La compañía utiliza la Relación Total de Accidentes Recordables (TRIR) como indicador de gestión para medir y gestionar el cumplimiento de sus resultados en accidentalidad. Se debe entender este indicador como el número de Accidentes Recordables o Registrables Acumulados sobre la cantidad de Horas Hombre Trabajadas (HHT) acumuladas en el mismo periodo, multiplicada por la constante 200.000. 
Según la relación con respecto a otros referentes teóricos uno de los riesgos identificados con mayor significancia dentro del sector de hidrocarburos especialmente en la fase de perforación es el sufrir lesiones ocasionadas por objetos que caen desde diferente altura, de los cuales el grado de afectación va relacionada con el tamaño de objeto que cae y con la altura de la caída del objeto.

La causa básica o raíz de mayor selección durante la caracterización realizada fue la de supervisión, liderazgo y/o planificación, seguida de procedimientos/ estándares de trabajo inadecuado y la ingeniería, diseño o mantenimiento inadecuado. Lo que indica que se debe trabajar muchos más en los comportamientos de las personas que en las condiciones del sitio e trabajo, sin descuidar lo segundo.

A nivel mundial el tema en estudio ocupa el tercer puesto de causalidad de accidentes en la construcción de la edificaciones, las cuales generan incapacidades y en Colombia la caída de objetos comparado con el sector de la construcción según el instituto de seguros sociales en el año 2005 reporto que dentro de los riesgos más significativos a los que están expuestos los operarios en una obra son la caída de materiales ocupando este un $15 \%$ de la evaluación de los riesgos ocupacionales; lo que indica que este tipo de riesgo se presenta en todos los sectores productivos

\section{AGRADECIMIENTOS}

Los autores reconocen reconocimientos a la Universidad Cooperativa de Colombia por facilitar el acceso al conocimiento a través de la investigación.

A Carlos Humberto Narváez Bohórquez, autor del estudio Caracterización de accidentes de trabajo causado por caídas de objetos en la empresa San Antonio Internacional durante los años 2010 y 2011.

\section{REFERENCIAS}

[1] Betancourt, B. (1984). Decreto 614 de 1984. Por se determinan las bases para la organización y administración de salud ocupacional en el país. Consultado Mayo 23 2012. Disponible en la página: http://copaso.upbbga.edu.co/legislacion/decreto_61 4\%2084\%200rganizacion\%20y\%20Administracion $\% 20$ Salud\%20Ocupacional.pdf

[2] González M. (2005), El sector de la construcción características y riesgos. Disponible en la página: www.laseguridad.ws/consejo/consejo/htlm/pys/pys3 02articulo1.doc.

[3] Instituto de estudios de estratégicas y estadísticas (2010) Condiciones de vida y de trabajo en la actividad petrolera en la provincia de Neuquen.

[4] Del Castillo, H. (2010) Procedimiento 02-01-107. Alertas de seguridad y alertas tempranas. Fecha de emisión 01 octubre 2010. Revisión 6. Consultado Mayo 23 2012. Disponible en la página: https://qhse.sanantoniointernacional.com/Corp/Doc umentos/Documentos\%20espaol/02\%20SISTEMA \%20DE\%20CALIDAD,\%20SALUD,\%20SEGURIDA
D\%20Y\%20AMBIENTE/02-

01\%20SEGURIDAD/Procedimientos/02-01-

$107 \% 20$ Alertas $\% 20$ de $\% 20$ Seguridad\%20y\%20Alert as\%20Tempranas.pdf

[5] Del Castillo, H. (2012) Procedimiento 02-01-109. Reporte e investigación de incidentes. Fecha de emisión 17 enero 2012. Revisión 10. Consultado Mayo 232012 . Disponible en la página:https://qhse.sanantoniointernacional.com/C orp/Documentos/Documentos\%20espaol/02\%20SI STEMA\%20DE\%20CALIDAD,\%20SALUD,\%20SE GURIDAD\%20Y\%20AMBIENTE/02-

01\%20SEGURIDAD/Procedimientos/02-0109\%20Reporte\%20e\%20Investigacion\%20de\%20A ccidentes.pdf

[6] Del Castillo, H. (2012) Procedimiento 02-01- 538. Programa de prevención de caída de objetos. Fecha de emisión 30 enero 2012. En Revisión.

[7] Cabanzo C, Catalina L, (2006) Diseño de un programa para la prevención de caída de objetos en taladros de perforación. Consultado Agosto 23 2014:

www.laseguirdad.ws/consejo/consejo/html/cis_ccs/ pdf/uagraria_01.pdf

[8] Drops online (2012). The Dropped Objects Prevention Scheme. Consultado Mayo 262014. Disponible en la página: http://www.dropsonline.org/index.asp? $i d=1 \&$ reflD $=4$ \&refID2=65\&contentID=65

[9] Forero de Saade, M. (1089). Resolución 1016 de 1989 "Por la cual se reglamenta la organización, funcionamiento y forma de los programas de salud ocupacional que deben desarrollar los patronos 0 empleadores en el país. Consultado Mayo 232012. Disponible en la página: http://www.alcaldiabogota.gov.co/sisjur/normas/Nor ma1.jsp?i=5412.

[10] Galilei. G. (1632). Biografía y vidas. Principio de la relatividad del movimiento. Consultado Junio 18 2014. Disponible en la página: http://www.biografiasyvidas.com/biografia/g/galileo. htm

[11] Gaviria. C. (1994). Decreto ley 1295 de 1994. Por el cual se determina la organización

[12] Guía Técnica Colombiana. GTC 45 (2010). Guía para la identificación de los peligros y la valoración de los riesgos en seguridad y salud ocupacional. Primera Actualización. Editada Enero 2011. Consultada Mayo 23 2014. Disponible en la página: http://so179.files.wordpress.com/2011/10/gtc_45.pd $f$

[13] Latin America Drilling Safety (2012). Asociación Latinoamérica para la seguridad en perforación Capitulo Colombia. Alertas HSE. Consultado Mayo 23 2014. Disponible en la página: http://www.latdrillingsafety.org/econtent/library/docu ments/DocNewsNo570DocumentNo956.pdf

[14] Palacio, D. (2008). Resolución 3673 de 2008. Por la cual se estable el reglamento técnico de trabajo seguro en alturas. Consultado Octubre 15 de 2014. Disponible en la página: https://www.minsalud.gov.co/Normatividad/RESOL UCI\%C3\%93N\%203673\%20DE\%202009.pdf 
[15] Pardo, R, (2012) Resolucion 1562 de 2012. Por la cual se modifica el sistema de riesgos laborales y se dictan otras disposiciones en materia de salud ocupacional. Consultado en Agosto 15 de 2014. Disponible en la página: http://wsp.presidencia.gov.co/Normativa/Leyes/Doc uments/ley156211072012.pdf

[16]J. F. Fuller; E. F. Fuchs and K. J. Roesler. "Influence of harmonics on power distribution system protection". IEEE Trans. Power Delivery, Vol. 3, No. 2, pp. 549-557, Apr. 1988.

[17]E. H. Miller. "A note on reflector arrays". IEEE Trans. Antennas Propagat., to be published.

[18] E. Clarke. Circuit Analysis of AC Power Systems. New York: Wiley, 1950, p. 81.

[19] G. O. Young. Synthetic structure of industrial plastics. In J. Peters (Ed.) Plastics, New York: McGraw-Hill, 1964, pp. 15-64.

[20] E. E. Reber; R. L. Mitchell and C. J. Carter. Oxygen absorption in the Earth's atmosphere. Aerospace
Corp., Los Angeles, CA, Tech. Rep. TR-0200 (4230-46)-3, Nov. 1968.

[21]D. Ebehard and E. Voges. "Digital single sideband detection for interferometric sensors". Presented at the 2nd Int. Conf. Optical Fiber Sensors, Stuttgart, Germany, PP. 34-42, 1984.

[22] J. L. Alqueres and J. C. Praca. "The Brazilian power system and the challenge of the Amazon transmission". Proc. 1991 IEEE Power Engineering Society Transmission and Distribution Conf., Madrid, Spain, pp. 315-320, 1991.

[23] K. M. Rahman. "Design and control of switched reluctante motor for electric and hybrid electric vehicle application". Ph.D. dissertation, directed by H. A. Toliyat and M. Ehsani, Texas A\&M University, Collage Station, Texas, Dec. 1998.

[24] Ley 9 de 1979, "Por la cual se dictan Medidas Sanitarias". http://www.alcaldiabogota.gov.co/sisjur/normas/Nor ma1.jsp?i=1177. 